\title{
Capturing Dynamics in Liquids with High-Speed CMOS Cameras - Opportunities and Challenges
}

\author{
See Wee Chee ${ }^{1,2,3}$, Abhik Datta ${ }^{1,2}$, Utkarsh Anand ${ }^{1,2,3,4}$, Duane Loh $^{1,2}$ and Utkur Mirsaidov ${ }^{1,2,3,4}$ \\ 1. Department of Physics, National University of Singapore, Singapore 117551 \\ 2. Centre for Bioimaging Sciences and Department of Biological Sciences, National University of \\ Singapore, Singapore 117557 \\ 3. Centre for Advanced 2D Materials and Graphene Research Centre, National University of Singapore, \\ Singapore 117546 \\ 4. NUSNNI-NanoCore, National University of Singapore, Singapore 117411
}

The development of liquid cell transmission electron microscopy (TEM) has allowed us to capture a range of phenomena in liquid samples, such as nanoparticle dynamics, electrochemical reactions and the biological structures within the microscope at high spatial resolution [1]. In principle, these studies should benefit from the capabilities of direct electron detection CMOS cameras, with their higher frame rates and higher sensitivity to electrons. State-of-the art cameras are capable of frame rates up to more than 1000 frames per second (sub-millisecond time resolution). For example, they enabled the capture of Pt nanoparticle nucleation events with atomic resolution at 400 frames per second [2].

In this paper, we describe current work at the Center of Biolmaging Sciences, National University of Singapore where the dynamics of nanoparticles are tracked with such cameras. Figure 1 and 2 show examples of this work where we looked at the coalescence of Au nanoparticles and rotational dynamics of cetyltrimethylammonium bromide (CTAB)-capped Au nanocubes respectively. To have sufficient signal-to-noise in the individual frames, we can either increase the electron flux on the liquid sample or decrease the frame rate. In this case, we opted to work with lower frame rates (35 to 100 frames/s) and limit the electron flux. Energy filtering with the in-column filter in our JEOL 2200FS microscope was also used to mitigate chromatic blurring in these liquid cells. In the coalescence study, we could follow events with lattice resolution of roughly $2 \AA$ in thin liquid layers and nanodroplets. On the other hand, the Au nanocubes were dispersed in 400-500 nm thick water, which lead to degradation of the image resolution. Previously, we reported that these Au nanocubes undergo in-plane rotation under the electron beam [3]. Here, we retained more free CTAB molecules in solution by reducing the dilution of the nanoparticle solution and we can see that the nanocubes presented themselves as hexagons in the TEM images. We believe that these adsorbed nanocubes had pivoted on their corners to reduce the electrostatic repulsion between the positively charged CTA ${ }^{+}$ligands on the (100) side facets of nanocube and the silicon nitride membrane, which gains a positive charge under the electron beam.

Despite the improvements offered by these high-speed cameras, electron fluxes on the order of $10^{2}$ to $10^{3} \mathrm{e}^{-/} \AA^{2} \cdot \mathrm{s}$ are typically required to obtain the clear images in a single frame at high magnification. Such conditions are untenable for imaging beam sensitive specimens or minimizing electron beam effects on the observed phenomena. Hence, the imaging parameters need to be optimized to keep the electron flux within reasonable limits while maintaining high frames rates. We will discuss the protocols that we are currently adopting for these liquid cell experiments. We are also developing computational methods to process noisy image sequences, so that we can capture the phenomena of interest while working at the finest temporal resolution and minimal electron fluxes. 


\section{References:}

[1] Frances M. Ross, Science 350 (2015) aaa9886.

[2] H.G. Liao et al., Science 345 (2014), p. 916.

[3] S.W. Chee et al., J. Phys. Chem. C 120 (2016), p. 20462

[4] The authors acknowledge the use of the facilities at the Center for BioImaging Sciences.

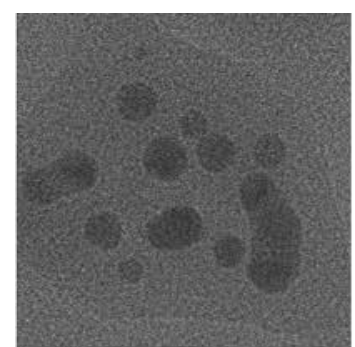

$-5 \mathrm{~nm}$

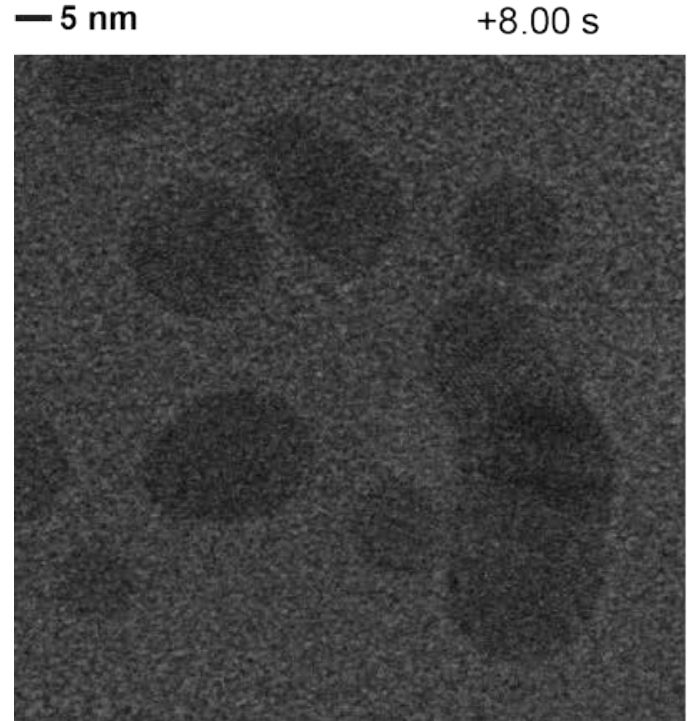

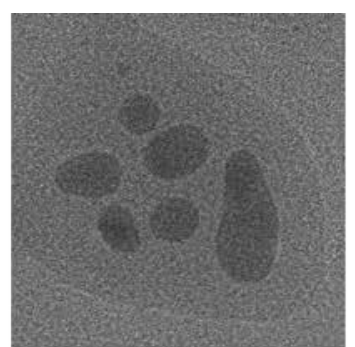

$+8.00 \mathrm{~s}$

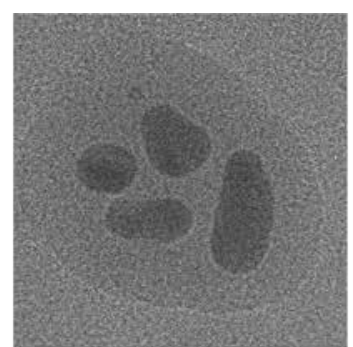

$+16.00 \mathrm{~s}$

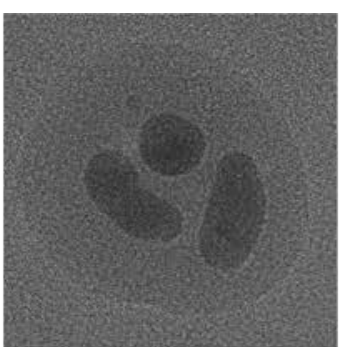

$+24.00 \mathrm{~s}$

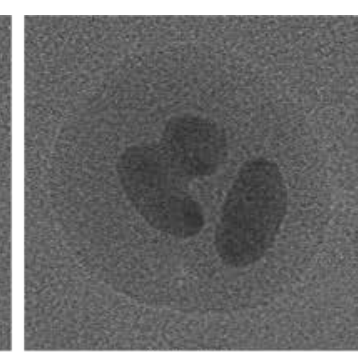

$+36.00 \mathrm{~s}$

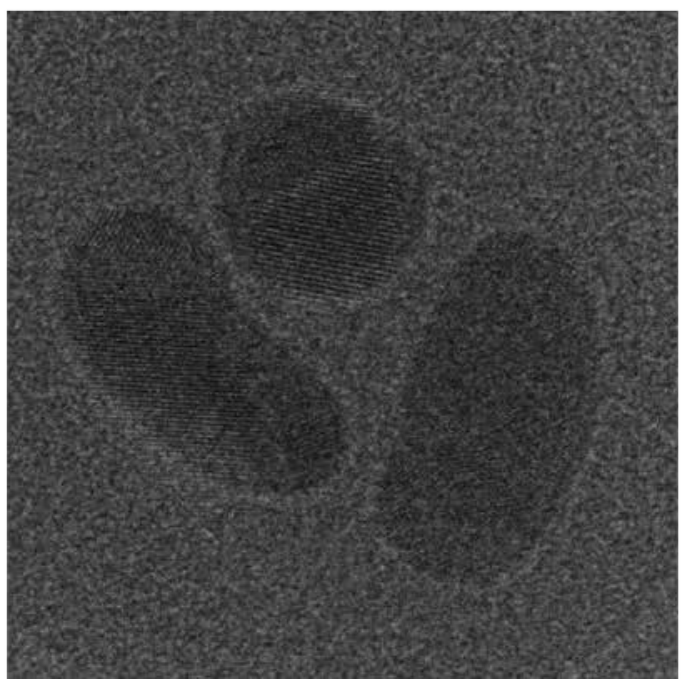

$5 \mathrm{~nm}$

Figure 1. The upper panel shows an image sequence depicting the coalescence of Au nanoparticles in a water droplet recorded at 80 frames per second. The lower panel shows two select images where the nanoparticles are captured with lattice resolution. These images were acquired with zero-loss filtering.

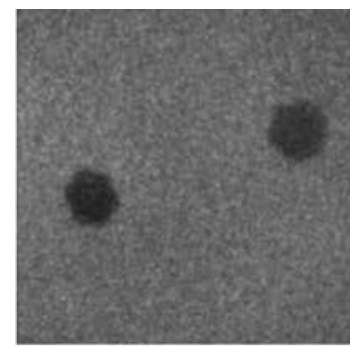

$50 \mathrm{~nm}$

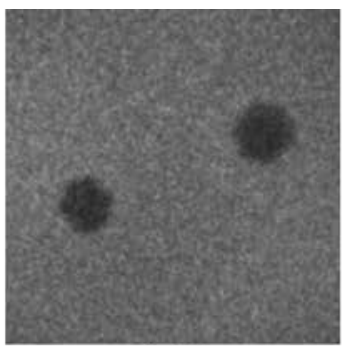

$+4.00 \mathrm{~s}$

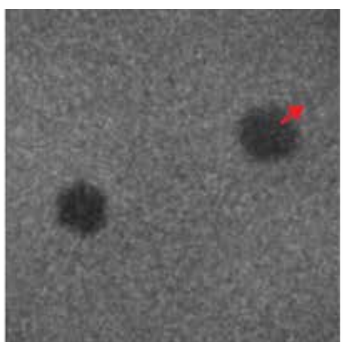

$+4.74 \mathrm{~s}$

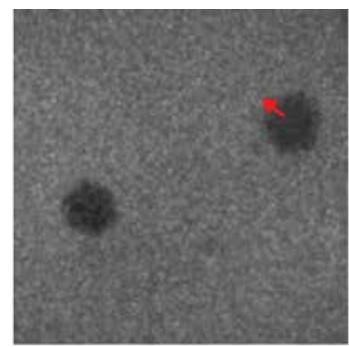

$+4.83 \mathrm{~s}$

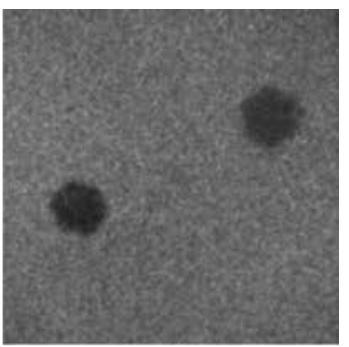

$+4.91 \mathrm{~s}$

Figure 2. Au nanocubes ( $20 \mathrm{~nm}$ in size) dispersed in a $400-500 \mathrm{~nm}$ thick water layer recorded at 35 frames per second. The nanocubes appear as hexagons in TEM image sequence. We can see that both nanocubes rotate between frames. Small displacements of the nanocube on the right are highlighted with red arrows. These images were acquired with most-probable loss filtering. This series of images had been processed with Gaussian blur filter with $\sigma=2$. 